\title{
Modes of Molecular Motion in Low Molecular Weight Polystyrene
}

\author{
C. M. Roland,,,† K. L. Ngai, ‘ and D. J . Plazek ${ }^{\ddagger}$ \\ Naval Research Laboratory, Washington, D.C. 20375-5342, and Department of Material Science and \\ Engineering, University of Pittsburgh, Pittsburgh, Pennsylvania 15261
}

Received March 3, 2004; Revised Manuscript Received J une 28, 2004

\begin{abstract}
The variations with temperature of the viscosity, $\eta$, and the relaxation times for various low molecular weight, unentangled polystyrenes (PS) are analyzed. We find that the temperature dependence of $\eta$ does not directly reflect the behavior of the global chain modes. Depending on molecular weight and temperature, $\eta$ can exhi bit a stronger temperature dependence than even the local segmental modes. However, this is only a consequence of the strong temperature dependence of the recoverable compliance J s. The Rouse relaxation time, $\tau_{1}$, deduced from the product of the viscosity and the recoverable compliance, has the expected behavior. For example, this Rouse time for a PS of mol ecular weight equal to $2 \mathrm{~kg} / \mathrm{mol}$ has the same temperature dependence as that for self-diffusion; hence, there is no enhancement of translational diffusion in the polymer. The same conclusion was reached by U rakawa et al. [U rakawa, O.; Swallen, S. F.; Ediger, M. D.; von Meerwall, E. D. Macromolecules 2004, 37, 1558], although the analysis leading to it was different. The conclusion is consistent with two extant explanations of the enhancement of translational diffusion in small molecular glass-formers, one which invokes spatially heterogeneous dynamics and the other ascribing the effect to the different coupling parameters for the translational and rotational correlation functions.
\end{abstract}

\section{Introduction}

Characterizing the dynamics of polymer chains is the sine qua non for understanding and predicting most of their physical properties. The gigantic size of polymer molecules implies an enormous number of degrees of freedom, which in turn gives rise to motion encompassing many decades of time over a correspondingly broad range of length scales. The usual approach to unraveling the viscoelasticity of polymers is to express the relaxation and transport dynamics in terms of modes, whose summation yields the macroscopic behavior. The starting point for analysis of the low-frequency motions is the Rouse model, generalized for undiluted polymer. According to the Rouse model, the chain is approximated as a collection of submolecule units ("beads"), connected by linear springs, with a friction coefficient $\zeta$. The chain motions are described in terms of a superposition of independent modes, each having a relaxation time given by ${ }^{1,2}$

$$
\tau_{\mathrm{p}}=\frac{\mathrm{b}^{2} \mathrm{~N}^{2} \zeta}{3 \pi^{2} \mathrm{kTp}}
$$

where $\mathrm{N}$ is the number of segments in the chain and $\mathrm{b}$ is the Kuhn step length. The $p=0$ mode measures the position of the center of mass; thus, the translational relaxation time for Rouse chains is related inversely to the self-diffusion constant, $\mathrm{D}^{2}$

$$
\tau_{0}=\frac{\left\langle r^{2}\right\rangle}{3 \pi^{2} D}
$$

where $\left\langle r^{2}\right\rangle$ is the mean-square end-to-end chain length. The terminal Rouse relaxation time $(p=1)$ can be evaluated from the relaxation modulus, $\mathrm{G}(\mathrm{t})$

\footnotetext{
† Naval Research Laboratory.

‡ University of Pittsburgh.
}

$$
\tau_{1}=\left(15 / \pi^{2}\right) \int_{0}^{\infty} \mathrm{tG}(\mathrm{t}) \mathrm{dt} / \int_{0}^{\infty} \mathrm{G}(\mathrm{t}) \mathrm{dt}
$$

It is proportional to the product of the zero-shear viscosity, $\eta$, and the equilibrium recoverable compliance, $\mathrm{J} \mathrm{s}^{1}$

$$
\tau_{1}=\left(15 / \pi^{2}\right) \eta \mathrm{J}_{\mathrm{s}}
$$

$\mathrm{J}_{\mathrm{s}}$, a measure of the elastic deformation during steadystate flow, is also related to the relaxation modulus

$$
\mathrm{J}_{\mathrm{s}}=\int_{0}^{\infty} \mathrm{tG}(\mathrm{t}) \mathrm{dt} /\left(\int_{0}^{\infty} \mathrm{G}(\mathrm{t}) \mathrm{dt}\right)^{2}
$$

whereby from eq 4

$$
\eta=\int_{0}^{\infty} \mathrm{G}(\mathrm{t}) \mathrm{dt}
$$

At temperature sufficiently higher than the glass temperature $T_{g}$, the Rouse model accurately predicts $\mathrm{s}_{\mathrm{s}}{ }^{1,3}$ Since J s varies only very weakly with temperature, $\tau_{0}$, $\tau_{1}$, and $\eta$ have essentially the same temperature dependence. However, as $T_{g}$ is approached by cooling, there is a marked decrease in the recoverable compliance, ${ }^{3}$ with a concomitant decrease in the quantity $\tau_{1} / \eta$.

The submolecule itself ( $p=N$ mode) must be sufficiently long to exhibit Gaussian statistics for its endto-end distribution. Dynamics transpiring on a smaller length scale (faster than $\tau_{\mathrm{N}}$ ) are thus referred to as subRouse modes. ${ }^{3,4}$ At sufficiently short range, only a few backbone bonds are involved, and this comprises the local segmental motion (dielectric $\alpha$-process) associated with the glass transition (structural relaxation). Williams ${ }^{5}$ proposed for polystyrene that five repeat units are required for the submolecule to exhibit Gaussian behavior. If this estimate is correct, then the Rouse model and eq 4 are rel evant for the description of chain relaxation for all the molecular weights of polystyrene discussed in this work. In the event that the molecular weight is too small and the sub-Rouse modes replace 
the Rouse modes, eq 4 still gives a value proportional to the terminal sub-Rouse relaxation time.

Resolving the various modes of motion from experimental data is difficult. For polymers lacking a dipole moment parallel to the chain axis, the normal modes are dielectrically inactive, whereby the local segmental relaxation ( $\alpha$-process) would be isolated in the dielectric relaxation spectrum. This is not the case for mechanical measurements, in which the response from the chain modes overlaps the local segmental process. This manifests in the well-known breakdown of time-temperature superpositioning in the transition zone of the viscoelastic spectrum of polymers. ${ }^{3}$ Read $^{6}$ and Inoue and coworkers ${ }^{7,8}$ have proposed a method of extracting the segmental ("G-component") and chain mode ("R-component") contributions by combining mechanical and birefringence measurements. The underlying assumption of the method is that the various modes are additive in the stress. This assumption is at odds with various experimental facts, as detailed el sewhere. 9,10

The present work is motivated by a recent publication by Urakawa et al., ${ }^{11}$ in which relaxation and transport properties of unentangled polystyrene (PS2K, molecular weight $=2 \mathrm{~kg} / \mathrm{mol}$ ) were reported. In their analysis, U rakawa et al. ${ }^{11}$ identified the terminal viscosity with the G-component of I noue et al..$^{8}$ and reported that $\eta_{0} / T$ had the same T dependence as the segmental dynamics. This is surprising since the viscosity is measured at low frequencies, long past the softening zone of the viscoelastic spectrum and the "G-component". In fact, according to the Rouse model, $60 \%$ of the viscosity of an unentangled polymer comes from the terminal Rouse mode. ${ }^{1}$ Since all Rouse modes have the same friction coefficient, all Rouse modes, as well as the viscosity, will have the same temperature dependence. On the other hand, segmental relaxation, which defines the glass transition, represents motion at length scales smaller than the shortest Rouse mode. Common practice is to define a segmental relaxation time, $\tau_{\text {seg, }}$ as the inverse of the frequency of the loss modulus maximum occurring near the glass transition. In terms of the relaxation modulus

$$
\tau_{\text {seg }}=\int_{0}^{\infty} \sin \left(\mathrm{t} / \tau_{\text {seg }}\right) \mathrm{tG}(\mathrm{t}) \mathrm{dt} / \int_{0}^{\infty} \cos \left(\mathrm{t} / \tau_{\text {seg }}\right) \mathrm{G}(\mathrm{t}) \mathrm{dt}
$$

It is often assumed that segmental relaxation and the Rouse modes are governed by the same monomeric friction coefficient and hence have the same timetemperature shift factors. Experimentally, this is found to be true only at high temperatures.1,3,12 At lower temperatures, the Rouse model prediction for $\mathrm{J} s$ breaks down, and the segmental relaxation time varies more with temperature than do the Rouse modes.

Herein we analyze existing data on unentangled PS (molecular weights in the range from 2 to $13 \mathrm{~kg} / \mathrm{mol}$ ) to compare the respective temperature dependences of the Rouse relaxation times, the viscosities, and the segmental relaxation times. We find, in agreement with Urakawa et al., ${ }^{11}$ that the Rouse and translational relaxation times (the latter obtained from diffusion measurements) have the same temperature dependence. However, particularly at lower temperatures, the viscosity exhibits a stronger $\mathrm{T}$ dependence, consistent with the decreasing magnitude of $\mathrm{J}_{\mathrm{s}}$. Quantities involving chain modesRouse relaxation times and diffusion coefficients-vary less with temperature than does the local segmental relaxation.

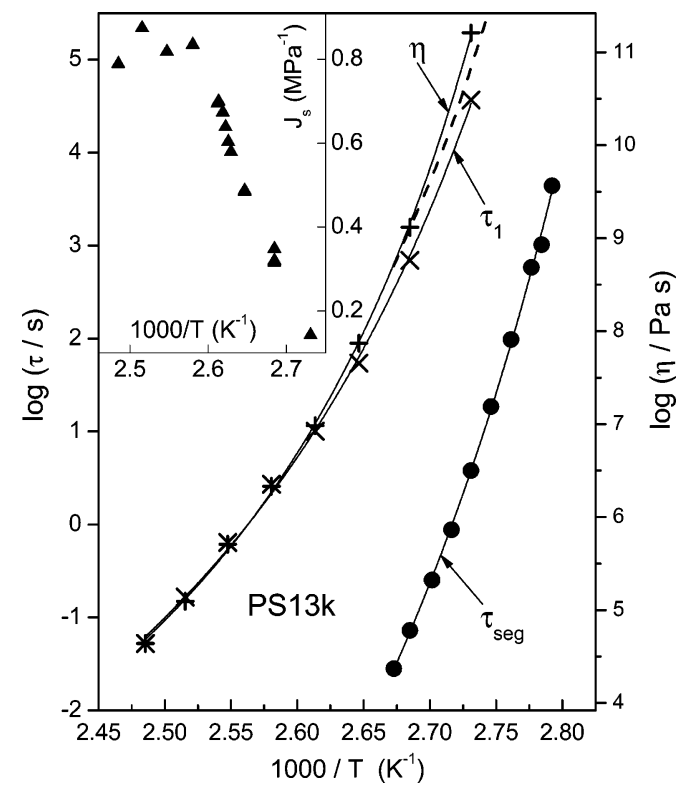

Figure 1. Arrhenius plot of Rouse relaxation times calculated using eq $4(x)$, steady-state creep viscosities (+), and segmental relaxation times $(\mathbf{0})$ for PS13K. ${ }^{13}$ The dotted line represents $\tau_{\text {seg }}$ shifted to coincide with $\eta$ at higher T. Solid lines represent Vogel-Fulcher-Tammann-Hesse (VFTH) fits. ${ }^{1}$ The respective ordinate scales have been adjusted to superpose $\tau_{1}$ and $\eta$ at the highest temperatures. The data were obtained by dynamic mechanical and shear rheometry measurements. The inset shows the steady-state recoverable compliance. ${ }^{13}$

\section{Results}

In Figure 1 are shown the local segmental relaxation times (-, defined as the inverse of the circular frequency of the maximum in the loss modulus) and the viscosities (+) for $\operatorname{PS} 13 K^{13}\left(\mathrm{M}_{\mathrm{w}}=13 \mathrm{~kg} / \mathrm{mol}\right)$, along with the relaxation times calculated for the first Rouse mode $(x)$, using eq 4 . The experimental values of $\mathrm{J}$ s are shown in the inset. A marked decrease in $\mathrm{J}$ s begins at $\tau_{1} \sim 10 \mathrm{~s}$. This change in $\mathrm{J}_{\mathrm{s}}$ amplifies the variation of $\eta$ with temperature (eq 4), so that $\eta$ exhibits a stronger T dependence than does $\tau_{1}$, as seen at lower temperatures in Figure 1. We have also shifted the segmental relaxations times (dashed line) to allow comparison to the viscosity. The variation of $\eta$ with temperature also exceeds that of $\tau_{\text {seg. }}$ Since the behaviors are all nonArrhenius over this range, the differences in temperature dependences will vary with temperature.

In Figure 2 are shown results similar to above, but for a lower molecular weight PS $\left(M_{\mathrm{w}}=3.4 \mathrm{~kg} / \mathrm{mol}\right) .{ }^{14,15}$ For PS3.4K, the decrease in J (shown in the inset) occurs at higher temperatures, for $\tau_{1}$ on the order of $10^{-3}$ $\mathrm{s}$. The consequence again is a stronger temperature dependence of $\eta$ in comparison to $\tau_{1}$. However, the local segmental relaxation times exhibit more variation with temperature than does $\eta$ or $\tau_{1}$, in contrast to the behavior of the PS13K in Figure 1. The stronger T dependence for the shorter time (involving shorter length scales) mode is the source of the well-known breakdown of time-temperature superpositioning in the glass transition zone. Thermorheological complexity is observed in that region of the viscoelastic spectrum because the relaxation times for the chain modes and segmental motion are sufficiently close that contributions from both mechanisms can be seen in isothermal viscoel astic measurements. Note that for $\mathrm{T}>385 \mathrm{~K}, \eta$ and $\tau_{1}$ have the same temperature dependence, consistent with the more nearly constant value of J s at higher 


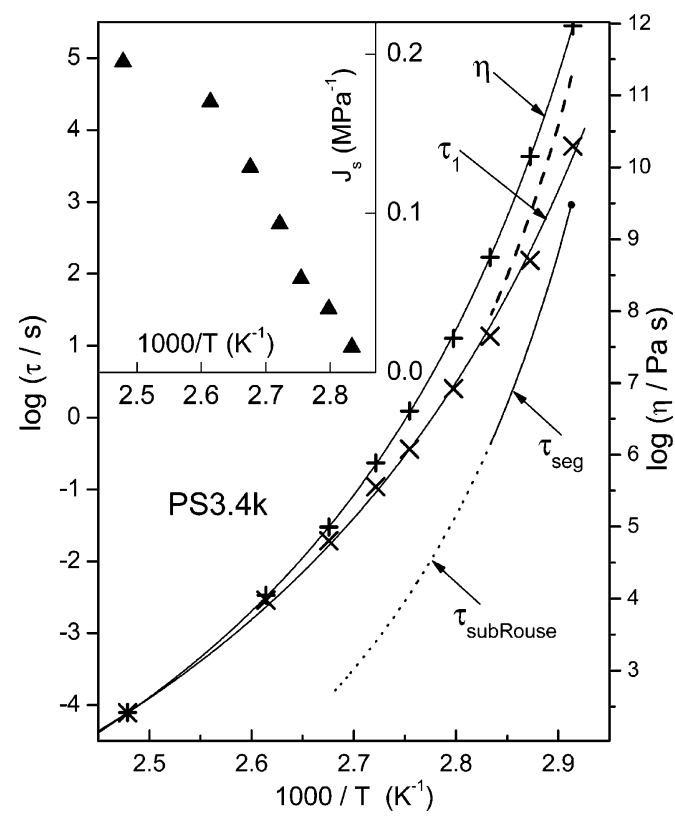

Figure 2. Arrhenius plot of Rouse relaxation times calculated from eq $4(x)$, steady-state creep viscosities $(+),{ }^{15}$ and segmental relaxation times ${ }^{14}$ (single point designated by $\bullet$, with solid line representing shift factors for higher temperatures) for PS4.3K. Dashed line extending from $\tau_{\mathrm{seg}}$ at higher temperatures represents shift factors for the sub-Rouse modes (see text). The dotted line represents $\tau_{\text {seg }}$ shifted to coincide with $\tau_{1}$ at higher T. Solid lines are VFTH fits to the respective data points. The respective ordinate scales have been adjusted to superpose $\tau_{1}$ and $\eta$ at the highest temperatures. The data were obtained from creep recovery experiments. ${ }^{15}$ The inset shows the steady-state recoverable compliance

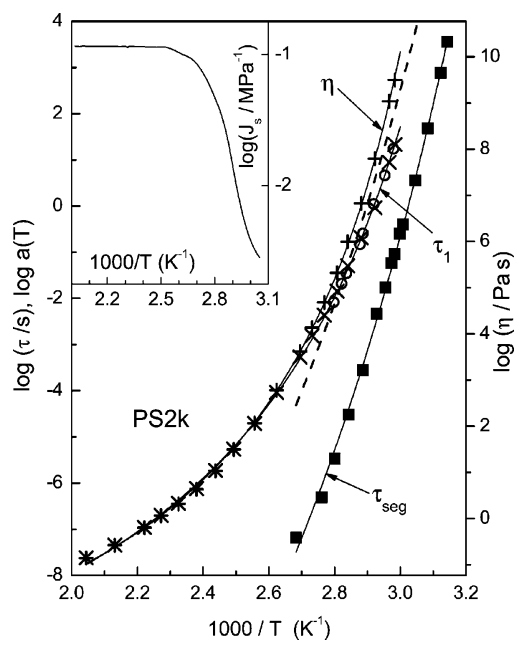

Figure 3. Arrhenius plot of the Rouse relaxation times $(x)$, local segmental relaxation times ${ }^{14}(\boldsymbol{\square})$, zero-shear rate dynamic viscosities $^{11}(+)$, and shift factors for the translational relaxation times calculated from the D in ref $11(O)$ for PS2K. The respective ordinate scales have been adjusted to superpose $\tau_{1}$ and $\eta$ at the highest temperatures. The solid line is the VFTH fit to $\tau_{\text {seg, }}$ and the dotted line is this same curve shifted to overlap the data for $\tau_{1}$. The inset shows the steady-state recoverable compliance.

temperatures. Also, for T > $350 \mathrm{~K}$, the creep compliance is no longer unambiguously dominated by the segmental modes, and thus we refer to these data as the sub-Rouse relaxation times.

In Figure 3 relaxation times ${ }^{14}$ and viscosities ${ }^{11}$ are plotted for a lower molecular weight polystyrene, PS2K $\left(\mathrm{M}_{\mathrm{w}}=2 \mathrm{~kg} / \mathrm{mol}\right)$. The steady-state recoverable compliance displayed in the inset was obtained by interpola- tion $\left(J_{s} \propto M_{w}\right)$ between published values ${ }^{15}$ for PS having molecular weights bracketing $2 \mathrm{~kg} / \mathrm{mol}$. The conspicuous decrease in J s corresponds to a value of $\tau_{1} \sim 6 \times 10^{-4} \mathrm{~s}$ at $\mathrm{T} \sim 370 \mathrm{~K}$. At temperatures below this, the viscosity $(+)$ varies more with temperature than does $\tau_{1}(x)$, while the curves for $\eta$ and $\tau_{\text {seg }}(\boldsymbol{\square})$ are roughly parallel. Again, the recoverable compliance contributes significantly to the temperature dependence of the viscosity.

Also included in Figure 3 are shift factors for the translational relaxation time, $\tau_{0}(O)$, obtained from the published ${ }^{11}$ self-diffusion coefficients for PS2K. The shift factors were chosen so that they overlap with the $\tau_{1}$ in Figure 3. This makes evident that the two Rouse relaxation times have essentially identical temperature dependences.

From eq 2, we can obtain a lower bound on $\left\langle\mathrm{r}^{2}\right\rangle$, which must be greater than the quantity $3 \pi^{2} \mathrm{D} \tau_{1}$, since $\tau_{0}>$ $\tau_{1}$. The result is $\left\langle r^{2}\right\rangle>290 \AA^{2}$. The mean-square endto-end length of a chain with i backbone bonds is also given by

$$
\left\langle\mathrm{r}^{2}\right\rangle=\mathrm{C}_{\mathrm{i}} \mathrm{i}^{2}
$$

where $C_{i}$ is the characteristic ratio, I $(=1.54 \AA)$ the carbon-carbon bond length, and $i=38$ for PS2K. The value of $C_{i}$ for PS2K is unavailable. Using the high $M_{w}$ limiting value of the characteristic ratio, $C_{\infty}=10.3 \AA$, provides an upper bound, so that we obtain $17 \AA<\left\langle r^{2}\right\rangle^{1 / 2}$ $<30 \AA$. This is consistent with the value reported by Abe et al., ${ }^{16}\left\langle r^{2}\right\rangle^{1 / 2}=25 \pm 2 \AA$, and thus supports our application of the Rouse model to polystyrene with $\mathrm{M}_{\mathrm{w}}$ $=2 \mathrm{~kg} / \mathrm{mol}$.

\section{Discussion and Summary}

From an analysis of various data for PS2K, Urakawa et al. ${ }^{11}$ concluded that the viscosity of low molecular weight PS is dominated by segmental motion, which they identified with the "glassy" or G-mode of I noue et al. ${ }^{17}$ The results in Figures $1-3$ show that this is not necessarily the case. There are small, but significant, differences in $\mathrm{T}$ dependences of $\tau_{\mathrm{seg}}$ and $\eta$, at least for the two higher molecular weight samples. The viscosity is dominated by the chain modes. At lower temperatures, approaching $T_{g}$, there is a decrease of the recoverable compliance, $J \mathrm{~s}$. This decrease is accompanied by an additional temperature dependence to $\eta$. Both reflect the variation of the relaxation modulus with $\mathrm{T}$ (eqs 5 and 6 ). A consequence is that the variation of $\eta$ with $\mathrm{T}$ becomes greater than the variation of $\tau_{1}$. The implication is that the viscosity may be a less useful quantity, at least when assessing temperature dependences of rheologically complex materials, including low molecular polymers at lower temperatures. For high polymers, and/or well-above $T_{g}$, there is no change of $\mathrm{J}_{\mathrm{s}}$ with temperature and hence no significant difference between the $T$ dependences of $\eta$ and $\tau_{1}$. Note that the decrease in I s for unentangled polymers at low temperature is contrary to the behavior expected from the pure Rouse model. The effect has been ascribed to the loss of the longer chain modes due to encroachment from local segmental relaxation. ${ }^{18}$ Most important herein is that eq 4 still gives a valid measure of the terminal relaxation time.

We show herein that for unentangled PS $\left(2 \leq \mathrm{M}_{\mathrm{w}}(\mathrm{kg} /\right.$ $\mathrm{mol}) \leq 13$ ) the T dependence of $\eta$ can be less than, equal to, or even greater than the variation of the local segmental relaxation times with temperature. The 


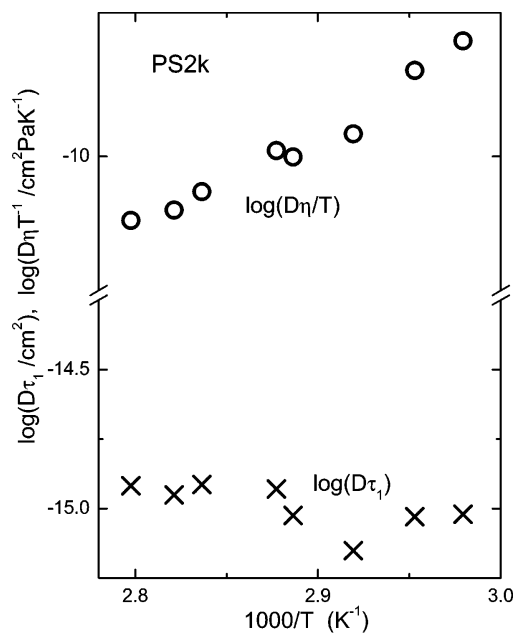

Figure 4. Product of the diffusion constant times the Rouse relaxation times $(x)$ and the diffusion constant times the viscosity divided by $\mathrm{T}(\mathrm{O})$ for PS2K.

behavior is reflected in the temperature variation of $\mathrm{J}$ s. The viscosity is a low-frequency, global quantity, which cannot be identified with either local segmental motion or the "glassy mode" of I noue et al. ${ }^{8} \tau_{1}$ is the "terminal" Rouse time for the reorientation of the chain end-toend vector, and it, as well as $\tau_{0}$, may be less affected by the temperature variations of $\mathrm{G}(\mathrm{t})$, in comparison to the steady-state compliance (viz. eqs 3 and 4). Together with the fact that that $\tau_{1}$ and $\tau_{0}$ are both Rouse relaxation times, this leads immediately to the conclusion that $\tau_{1}$ has the same $T$ dependence as $\tau_{0}$.

In Figure 4, the product of $\tau_{1}$ and the $D$ from ref 11 are plotted vs temperature for PS2K. There is no significant variation, supporting a principal finding of Urakawa et al. ${ }^{11}$ that $\mathrm{D}\left(\propto \tau_{0}{ }^{-1}\right)$ has the same temperature dependence as the terminal (longest Rouse) relaxation time. This conclusion is reached herein directly from experimental viscoelastic quantities (i.e., $\eta$ and $\mathrm{J}$ s), without recourse to the R-mode analysis of Inoue et al. ${ }^{8}$ As has been discussed el sewhere,, 10 the deconvolution of the dynamic modulus into distinct glassy and rubbery components, ${ }^{7}$ originally proposed by Read, ${ }^{6}$ relies on a stress-additivity assumption which is questionable. ${ }^{19,20}$

Also shown in Figure 4 is the quantity $\mathrm{D} \eta / \mathrm{T}$ (the viscosity is divided by temperature in accord with the Stokes-Einstein rule). This quantity increases with decreasing temperature, a manifestation of the wellknown enhancement of translational motion near $\mathrm{T}_{\mathrm{g}}{ }^{11}$ Our analysis herein obviates the need to invoke the argument that dynamic heterogeneity of segmental relaxation is averaged out due to the larger size of the PS2K molecule relative to the length scale for spatial heterogeneity. ${ }^{11}$ While this argument is needed to rationalize the absence of enhancement of translation diffusion in PS2K in comparison to the terminal relaxation rate $1 / \tau_{1}$ (corresponding to $1 / \tau_{\text {term }}$ in ref 11 ), the present approach arrives at the same conclusion directly. Spatially heterogeneous dynamics has been proposed $^{21}$ as the cause of the enhancement in smallmolecule glass-formers of translation diffusion compared to the rate of rotational relaxation. The inference of Urakawa et al. ${ }^{11}$ is that the PS2K molecules experience an average environment, which diminishes or even eliminates enhancement of their translation diffusion. Accordingly, the plausibility of the spatially heteroge-
Table 1. Activation Energies for Polyethylene and Hydrogenated Polybutadiene $\left(T \gg T_{g}\right)$

\begin{tabular}{ccc}
\hline & \multicolumn{2}{c}{$\mathrm{E}_{\mathrm{a}}(\mathrm{kJ} / \mathrm{mol})$} \\
\cline { 2 - 3 } variable & polyethylene & \multicolumn{1}{c}{ hydrogenated polybutadiene } \\
\hline$\eta$ & $26.6^{22}$ & $30^{24}$ \\
$\mathrm{D}$ & $22.9^{23}$ & $25.9^{25}$ \\
a 8\% vinyl content. &
\end{tabular}

neous dynamics explanation of enhanced translational diffusion for small molecular glass-formers ${ }^{21}$ is consistent with the absence of it in PS2K. However, this explanation in terms of heterogeneity length scales does not address the situation for polymers of high molecular weight. For entangled chains, the viscosity and selfdiffusion coefficient exhibit different temperature dependences. For example, as shown in Table 1, the activation energy for the viscosity of polyethylene ${ }^{22}$ is larger than $\mathrm{E}_{\mathrm{a}}$ for self-diffusion. ${ }^{23}$ The same is true for (the structurally similar) hydrogenated polybutadiene. ${ }^{24,25}$ These molecules, of course, are larger in size than PS2K, and so the putative averaging of spatial heterogeneities still occurs. However, the decoupling of $\eta$ and $\mathrm{D}$ in high- $\mathrm{M}_{\mathrm{w}}$ polymers is observed at high temperatures, $T>T_{g}$, where dynamic heterogeneity is not expected to be an issue. Thus, an explanation based on spatially heterogeneous dynamics is not operative in this regime.

There is, however, an alternative hypothesis regarding the decoupling between translational diffusion and rotational motion observed in small molecules. One obvious difference is that $D$ involves the correlation of the center of mass (one point), while $\tau_{\mathrm{c}}$ is determined by the time correlation of the transition dipole of the molecule, $\bar{u}$, a vector. Thus, a priori, there is no reason to expect their correlation functions to be the same. Moreover, from the fact that the vector $\bar{u}$ is defined by two points in space, one expects intermolecular constraints to be more effective in slowing the rotation of $\bar{u}$ than the motion of the center of mass. The coupling model explanation of the decoupling is based on the difference in the correlation functions (and hence coupling parameters) for self-diffusion and rotation. ${ }^{26}$ ACcording to this approach, the same mechanism underlies the decoupling seen in small molecular liquids near $T_{g}$ and in entangled polymers at high temperature.

However, is the lack of this decoupling in PS2K consistent with this alternative explanation? The answer is in the affirmative and is easily arrived at as follows. As formulated, in the Rouse model for undiluted polymers, the relaxation modes of each Rouse chain are independent of those for other chains. Hence, by definition, all Rouse modes, including $\tau_{0}$ (self-diffusion) and $\tau_{1}$ (rotation) in unentangled PS, experience no intermolecular coupling, and thus their coupling parameters equal zero. ${ }^{27} \mathrm{M}$ oreover, since their coupling parameters are the same, it follows that $\tau_{0}$ and rotation $\tau_{1}$ have the same temperature dependence; ${ }^{26}$ there is no decoupl ing in PS2K, in accord with the finding of Urakawa et al. ${ }^{11}$ Although for Rouse chains near $T_{g}$, self-diffusion is not enhanced relative to terminal relaxation, for high molecular weight polymers, reorientation and self-diffusion are associated with different (nonzero) coupling parameters, again due to different correlation functions (reflecting different weighting of entanglement interactions). F rom the dependence of the relaxation time on the magnitude of the coupling parameter, the differ- 
ences in temperature dependences of viscosity and selfdiffusion (viz. the $E_{a}$ in Table 1 ) can be accounted for ${ }^{28}$

Acknowledgment. This work was supported by the Office of Naval Research. We thank G. C. Berry and M. D. Ediger for helpful comments.

\section{References and Notes}

(1) Ferry, J . D. Viscoelastic Properties of Polymers; Wiley: New York, 1980.

(2) Doi, M.; Edwards, S. F. The Theory of Polymer Dynamics; Clarendon: Oxford, 1986

(3) Ngai, K. L.; Plazek, D. J. Rubber Chem. Technol. 1995, 68, 376.

(4) Santangelo, P. G.; Ngai, K. L.; Roland, C. M. Macromolecules 1993, 26, 2682.

(5) Williams, M. L. J . Polym. Sci. 1962, 62, S7.

(6) Read, B. E. Polym. Eng. Sci. 1983, 23, 835.

(7) Inoue, T.; Okamoto, H.; Osaki, K. Macromolecules 1991, 24, 5670.

(8) Inoue, T.; Onogi, T.; Yao, M. L.; Osaki, K. J . Polym. Sci., Part B: Polym. Phys. 1999, 37, 389.

(9) Mott, P. H.; Roland, C. M. Macromolecules 1998, 31, 7095.

(10) Roland, C. M.; Mott, P. H. Macromolecules 1999, 32, 4728.

(11) Urakawa, O.; Swallen, S. F.; Ediger, M. D.; von Meerwall, E. D. Macromolecules 2004, 37, 1558.

(12) Roland, C. M.; Ngai, K. L.; Santangelo, P. G.; Qiu, X. H.; Ediger, M. D.; Plazek, D. J . Macromol ecules 2001, 34, 6159.
(13) Santangelo, P. G.; Roland, C. M. J . Rheol. 2001, 45, 583.

(14) Santangelo, P. G.; Roland, C. M. Macromolecules 1998, 31 4581.

(15) Plazek, D.J .; O’Rourke, V. M. J . Polym. Sci., Part A2: Polym. Phys. 1971, 9, 209.

(16) Abe, F.; Einaga, Y.; Yoshizaki, T.; Yamakawa, H. Macromolecules 1993, 26, 1884

(17) Inoue, T.; Osaki, K. Nihon Reoroji Gakkaishi 1999, 27, 251.

(18) Ngai, K. L.; Plazek, D. J .; Deo, S. S. Macromolecules 1987 20, 3047. Ngai, K. L.; Plazek, D. J .; Bero, C. A. Macromolecules 1993, 26, 1065.

(19) Plazek, D. J .; Echeverria, I. J . Rheol. 2000, 44, 831.

(20) Roland, C. M.; Archer, L. A.; Mott, P. H.; Sanchez-Reyes, J. J. Rheol. 2004, 48, 395.

(21) Cicerone, M. T.; Wagner, P. A.; Ediger, M. D. J . Phys. Chem. B 1997, 101, 8727.

(22) Mendelsohn, R. A.; Bowles, W. A.; Finger, F. L. J . Polym. Sci., Part A2 1970, 8, 105.

(23) Tirrell, M. Rubber Chem. Technol. 1984, 57, 523.

(24) Raju, V. R.; Rachapudy, H.; Graessley, W. W. J . Polym. Sci., Part B: Polym. Phys. 1979, 17, 1223.

(25) Bartels, C. R.; Crist, B.; Graessley, W. W. Macromolecules 1984, 17, 2702.

(26) Ngai, K. L. J . Phys. Chem. B 1999, 103, 10684.

(27) Ngai, K. L.; Plazek, D. J .; Rendell, R. W. Rheol. Acta 1997, 36, 307.

(28) McKenna, G. B.; Ngai, K. L.; Plazek, D. J . Polymer 1985, 26, 1651.

MA049573C 